OPEN ACCESS

Edited by:

Trine Bilde,

Aarhus University, Denmark

Reviewed by:

Pau Carazo,

University of Valencia, Spain

Seth Barribeau,

Swiss Federal Institute of Technology in Zurich (ETHZ), Switzerland

${ }^{*}$ Correspondence:

Sze H. Yek

szehuei.yek@unil.ch

${ }^{\dagger}$ These authors have contributed equally to this work.

Specialty section:

This article was submitted to

Social Evolution,

a section of the journal

Frontiers in Ecology and Evolution

Received: 17 March 2016

Accepted: 01 June 2016

Published: 15 June 2016

Citation:

Otani S, Bos N and Yek SH (2016)

Transitional Complexity of Social

Insect Immunity.

Front. Ecol. Evol. 4:69.

doi: 10.3389/fevo.2016.00069

\section{Transitional Complexity of Social Insect Immunity}

\author{
Saria Otani ${ }^{1+}$, Nick $\mathrm{Bos}^{2 \dagger}$ and Sze H. Yek ${ }^{3 * t}$ \\ ${ }^{1}$ Centre of Social Evolution, University of Copenhagen, Copenhagen, Denmark, ${ }^{2}$ Centre of Excellence in Biological \\ Interactions, University of Helsinki, Helsinki, Finland, ${ }^{3}$ Department of Ecology and Evolution, University of Lausanne, \\ Lausanne, Switzerland
}

Genomic analyses between insects are often conducted by comparing host genomes to that of Drosophila. For honey bees, this led to the claim that the evolutionary transition to eusociality resulted in a reduction of immunity-related genes. Although this claim pervades the literature, contradictory evidence exists. Many genomic studies, however, are not comparable due to methodological differences, and only focus on the physiological aspect of the immune system, thus potentially missing other immunity components. We advocate more comprehensive comparative studies, as well as the analysis of insect-associated defensive microbiotas to improve our understanding of the complexity of social insect immunity.

\footnotetext{
Keywords: gene annotation, immunity, comparative genomics, microbiota, social evolution, evolutionary transitions
}

\section{EUSOCIALITY AND IMMUNITY COMPLEXES}

As one of the major evolutionary transitions in animal societies, eusociality leads to fundamental changes, including increased complexity of communication systems as well as more frequent contacts between individuals (Bourke, 2011). This frequent contact between often highly related individuals provides optimal conditions for the spread of pathogens. In response to this, social insects have, next to their physiological, behavioral, and personal innate immune systems, an additional layer of defense compared to their solitary contemporaries: social immunity, which refers to the cooperative immunity functions that are mounted by a group of individuals to help combat disease threats (Cremer et al., 2007). For example, honey bees collecting resins to coat their hives (Simone et al., 2009) and metapleural gland secretions in leaf-cutting ants that are deployed against parasites that compete with their fungus gardens (Yek et al., 2012). Thus, in the transition from solitary to eusocial, one could expect major changes in the genetic and phenotypic traits involved in how organisms face parasitic threats. The distinction and manifestation of personal and social immunity had been reviewed extensively elsewhere (e.g., Cremer et al., 2007; Cremer and Sixt, 2009; Wilson-Rich et al., 2009; Cotter and Kilner, 2010). Here, we will focus our review on the consequences of social living on two components of immunity: genes related to innate immunity (Hoffmann, 1995), and defensive microbiota harbored by individuals.

The first thorough genomic comparison between social and solitary insects found that honey bees have a lower number of genes related to immunity compared to the fruit fly and mosquito; the only insect genomes available at the time (Evans et al., 2006). The authors argued that the emergence of social immunity could have reduced the expression of innate immunity due to investment tradeoffs between these two processes. Therefore, honey bees would not rely on innate immunity as much as solitary insects. However, more recent comparative genomic studies of multiple insect genomes across different levels of social organization, indicated that eusociality by itself does not 
appear to affect the number of immunity-related genes in hymenopteran ants and bees (Wurm and Keller, 2010; Smith et al., 2011; Simola et al., 2013; Roux et al., 2014; Barribeau et al., 2015; Grozinger and Robinson, 2015; Kapheim et al., 2015; Sadd et al., 2015) or isopteran termites (Terrapon et al., 2014; Korb et al., 2015), challenging the previous hypothesis of eusociality causing relaxed selection on innate immunity. While these further analyses have supplied evidence that eusociality by itself is not associated with the reduction in immune-gene counts, the old claim is still being propagated in the recent literature (e.g., Nish and Medzhitov, 2011; Gadau et al., 2012; Mattila et al., 2012; Schöning et al., 2012; Evison et al., 2013; Meunier, 2015).

Immune gene counts do not deliver the detailed description of insect defense mechanisms. Next to cellular and humoral defenses, other players have major roles in insect pathogen defense. These are external immune defenses such as antimicrobial secretions or insect-associated bacterial communities. The role of antimicrobial secretions has recently been reviewed (Otti et al., 2014), so we focus here on insect bacterial communities. Insect-associated bacteria can proliferate in or on the insect, such as in the gut, on the cuticle, or even intracellularly (Engel and Moran, 2013). Attention to this field has expanded enormously in the last 10 years with the advent of new affordable sequencing techniques (Engel and Moran, 2013), providing insights into the defensive mechanisms of insectmicrobial associations. For example, microbial communities can produce antimicrobial compounds that suppress potential pathogens in the insect, e.g., antibiotic-producing Actinobacteria in ants and termites (Currie et al., 1999; Mattoso et al., 2011; Visser et al., 2011), or can colonize several sites in or on the insect preventing infectious pathogens from being established, e.g., a number of highly abundant bacterial phyla occupy the majority of termite digestive tract leaving the remaining phyla in relatively low abundances (Otani et al., 2014). Finally, insect-associated microbes can modulate the host's immune system to enhance its efficiency against pathogens, e.g., Blochmannia bacterium can improve Camponotus ant immune responses (de Souza et al., 2009). If secretions or bacteria in these insect-associated communities are able to be shared among nestmates, for example through allogrooming or trophallaxis (Hamilton et al., 2011; Konrad et al., 2012), it is plausible that they would qualify as part of social immunity (see definition above), and hence the complex and intricate interactions between bacterial communities and their host immunity would change through the evolutionary transition from solitary to eusocial insects. To date, limited comparisons between solitary and social insect gut microbiotas have been conducted (Martinson et al., 2011; Dietrich et al., 2014; Otani et al., 2014), but none has investigated the differences in microbe-derived immunity between social and solitary insect microbiotas.

Here, we discuss the potential flaws of the earlier suggestions that eusociality affected the repertoire of immunity-related genes, and argue that eusocial insect immunity genes did not dramatically change from solitary ancestors. We then discuss the up-and-coming field of microbe-derived immunity and emphasize that gene- and microbe-level immunity should be investigated in parallel when studying the transitional gradients from a solitary to eusocial lifestyle, in order to form an integrated understanding of immunity as a whole. Finally, we recommend that (1) standardized approaches should be adopted for gene annotation and sequencing, (2) comparison of immunity should include multiple species representing different levels of sociality within a phylogenetic context, if possible, to capture transitional changes (e.g., Barribeau et al., 2015; Kapheim et al., 2015), and (3) functional experiments should be carried out to improve our understanding of differentially regulated genes of unknown function.

\section{MULTI-LEVEL -OMICS APPROACH ON SOCIAL IMMUNITY}

A major limitation in comparing immune systems across diverse insect taxa is that most immunity-related proteins have mainly been characterized in dipterans, with a large focus on Drosophila melanogaster. Thus, immunity-related gene annotations of social insects relied largely on homology similarities to dipteran DNA sequences when the first social insect genome was published in 2006. This approach misses two components of immunity-related genes: (i) host taxon-specific genes, which have been shown to exist in great numbers in Nasonia genomes (e.g., Sackton et al., 2013) and (ii) genes that are undergoing strong selection pressure, such as the peptidoglycan recognition proteins (PGRPs) and antimicrobial proteins (AMPs; Viljakainen et al., 2009), possibly obscuring previous homologies. These two features result in further divergence from the conserved component of immunity-related genes found in distant Drosophila genomes. Furthermore, genes that evolve de novo within taxa will also likely be missed by homology comparison to dipterans (Sackton et al., 2013).

To resolve the above issues, we suggest that researchers adopt a multi-level -omics approach, i.e., comparisons not only on the DNA sequence level but also on functional units such as protein domains and transcriptome. Protein analysis was carried out as a test case on the solitary wasp Nasonia vitripennis, whereby computational predictions based on known sequences and conserved protein domains found 146 immunity-related genes not identified in previous annotations, increasing the known immunity-related genes by $58 \%$ for this species (Brucker et al., 2012). Interestingly, as comprehensive as the analysis of Brucker et al. was, the authors only found $46 \%$ of immunity-related genes originally published. Thus, while individual annotation methods all have their advantages and drawbacks, using them in unison ensures more comprehensive results.

In addition to studying protein domains, RNA sequencing of immune-challenged individuals can provide a better estimate of immunity-related genes. For example, Yek et al. (2013) compared the transcriptome of infected and non-infected leaf-cutting ants (Acromyrmex echinatior) and found that a high number of genes responding to infection did not show any homology to the annotated genome of D. melanogaster and other insect genomes. While not all of these affected genes are predicted to be involved in immunity (as infection might affect a much wider scale of gene families), it highlights that for many genes in novel study systems, 
we currently have no clear functional identity. Given that genes involved in immune responses are expected to be consistently co-expressed when organisms are being challenged, this method is useful to narrow down candidate immunity-related genes, which then can be further targeted for functional tests such as RNAi. Complementing DNA-sequencing with protein and transcriptome studies will thus give a much more complete image of immune responses in insects.

Thorough comparison using such a multi-level -omics approach has rarely been conducted across the genomes of insects at different levels of social organization complexity. Therefore, to resolve the question of whether a transition to a eusocial lifestyle affects the number of immunity-related genes or not, future studies should focus on adopting multi-level omics comparisons. With the application of this method, it would be possible to properly investigate whether transition to eusociality brings about a reduction in immunity-related genes in bees (Wurm and Keller, 2010; Barribeau et al., 2015; Kapheim et al., 2015). In particular, Barribeau et al. compared immunityrelated genes from advanced eusocial, intermediate eusocial, and solitary bees (Libbrecht and Keller, 2015), and found comparable numbers of immunity-related genes between the bees regardless of the level of their sociality (Barribeau et al., 2015).

\section{SOCIAL INSECT MICROBIOTA CONTRIBUTIONS TO HOST DEFENSES}

A marked contribution to insect immunity is also provided by the involved microbial communities (Engel and Moran, 2013 and references therein). Due to the recent proliferation of insect microbiota studies, social insect gut microbial communities are evident to be more specialized and structured when compared to solitary insects (Sabree et al., 2012; Engel and Moran, 2013; Otani et al., 2014). Given these seemingly more specialized gut microbes, an appreciable number of defensive genes/peptides observed in the social insect genetic biome are of microbial origins (e.g., Koch and Schmid-Hempel, 2011; Engel and Moran, 2013). We focus our discussion on gut microbial communities (as opposed to microbes present in/on other parts of the insect body), because we postulate that these internally housed bacterial communities would have more intimate relationships with the innate immunity of their symbiont host due to the protected environment that they were in. Indeed, recent studies have provided insights into the complimentary roles of innate immunity and gut microbiota. For example, bacteria with antimicrobial and fungal cell wall degradation properties are found to be present in termite gut and nest microbiotas (Otani et al., 2014; Poulsen et al., 2014; Rosengaus et al., 2014) with higher abundances compared to their ancestral solitary cockroaches (Dietrich et al., 2014; Otani et al., 2014). In bees, a number of antimicrobial peptide-producing bacteria have been sequenced to show that they are selectively higher in abundance in bees compared to Drosophila (Wong et al., 2011 and references therein). Finally, gut bacterial species in honeybees, e.g., Snodgrasella alvi and Gilliamella apicola, have been reported to have protective roles against the trypanosomatid parasite Crithidia bombi (Koch et al., 2012; Cariveau et al., 2014; Moran, 2015), in contrast to the solitary bee genera where they are detected in low abundances (Martinson et al., 2011).

To our knowledge, no study has specifically investigated the variations between social and solitary insect gut microbes that are known to be immunity-related, even though this could help elucidate the role of microbiota-associated defenses in eusociality. Correlated studies on general characterisation of gut microbiota between social and solitary species reported distinctive gut microbiotas between solitary and social bee species and linked it to changes in the diet (Martinson et al., 2011). A recent study on chimpanzee directly links the increase in social interactions to the propagations of beneficial microbiota (Moeller et al., 2016), suggesting that similar processes could also play a role in the defensive microbiota of eusocial insects. As the transition from solitary lifestyle to eusociality often involve whole suites of adaptations, we have strong reasons to suspect defensive microbiota adapt differently according to host's lifestyle, and particularly to the risk of infection. This suggests that the transition from solitary to social living changes the composition of the host's microbiota, yet the detailed profiles of these changes, and how it affects the social immunity system, are to be investigated. However, with the recent expansion of high throughput sequencing, previous techniques such as 454 pyrosequencing are not accurately comparable to newer ones such as Illumina. The different databases used for classification in different methods also complicate comparisons. Finally, the challenges faced by microbiota metagenomics are similar to genomic comparisons. Here, we are also riddled with unknown functions assigned to the insect-associated bacteria. This is particularly evident in the rich bacterial communities of social insect guts, such as ants and termites (Hongoh, 2010) compared to those of solitary insects. However, other complimentary techniques, such as FISH (e.g., Sapountzis et al., 2015) and interactomes (i.e., transcriptomes of both insect host and microbiota) are able to resolve some of these challenges, and hence improve our understanding of microbiota-associated defenses inside their respective hosts.

\section{CONCLUSIONS}

While the claim that honey bees, and social insects in general, harbor fewer immunity-related genes than solitary insects currently pervade the literature, available evidence is contradictory. Although a recent paper showed that a negative correlation exists between the number of workers a species has (as a proxy for the level of sociality) and their encapsulation response (a simple measurement of an innate immune response; LópezUribe et al., 2016), other papers suggest that a possible reduction in immune genes is not due to eusociality itself (e.g., Barribeau et al., 2015), and that more comprehensive research is needed for a well-supported conclusion. Even though a comparison within a clade of social insect (bees; Barribeau et al., 2015) has been done, proper comparisons from solitary to social insects of different degrees of sociality, using standardized methods (annotation and sequencing) have not been conducted yet. Unfortunately, direct 
and detailed comparison studies between individual are usually not feasible, mainly because of the huge variation in the methods employed in the sequence analyses (Brenner, 1999 and references therein), for example a study that employed 454 pyrosequencing is not directly comparable to a study that used Illumina MiSeq sequencing (Kozich et al., 2013).

Therefore, when investigating immunity, we recommend not only comparing immunity-related genes, protein domains and the transcriptome of the studied insect (Bader et al., 2003), but striving to further include social immunity contributed defensive genes from the associated microbiota. This is because insects are likely to be complementing their defenses with microbes along with their own physiological pathways. Although this approach still faces limitations regarding genes which have no conserved domains and no homologs in other model species, we can significantly improve our understanding of the transitional complexity of social insect immunity in the future by integrating the multiple components (e.g., host genes and microbe-contributed genes) that form such defense system.

\section{REFERENCES}

Bader, G. D., Betel, D., and Hogue, C. W. V. (2003). BIND: the biomolecular interaction network database. Nucleic Acids Res. 31, 248-250. doi: 10.1093/nar/gkg056

Barribeau, S. M., Sadd, B. M., du Plessis, L., Brown, M. J., Buechel, S. D., Cappelle, K., et al. (2015). A depauperate immune repertoire precedes evolution of sociality in bees. Genome Biol. 16, 1-21. doi: 10.1186/s13059-015-0628-y

Bourke, A. F. G. (2011). Principles of Social Evolution. Oxford, UK: Oxford University Press.

Brenner, S. E. (1999). Errors in genome annotation. Trends Genetics 15, 132-133. doi: 10.1016/S0168-9525(99)01706-0

Brucker, R. M., Funkhouser, L. J., Setia, S., Pauly, R., and Bordenstein, S. R. (2012). Insect Innate Immunity Database (IIID): an annotation tool for identifying immune genes in insect genomes. PLOS ONE 7:e45125. doi: 10.1371/journal.pone.0045125

Cariveau, D. P., Elijah Powell, J., Koch, H., Winfree, R., and Moran, N. A. (2014). Variation in gut microbial communities and its association with pathogen infection in wild bumble bees (Bombus). ISME J. 8, 2369-2379. doi: 10.1038/ismej.2014.68

Cotter, S. C., and Kilner, R. M. (2010). Personal immunity versus social immunity. Behav. Ecol. 21, 663-668. doi: 10.1093/beheco/arq070

Cremer, S., Armitage, S. A. O., and Schmid-Hempel, P. (2007). Social immunity. Curr. Biol. 17, R693-R702. doi: 10.1016/j.cub.2007.06.008

Cremer, S., and Sixt, M. (2009). Analogies in the evolution of individual and social immunity. Philos. Trans. R. Soc. B 364, 129-142. doi: 10.1098/rstb.2008.0166

Currie, C. R., Scott, J. A., Summerbell, R. C., and Malloch, D. (1999). Fungusgrowing ants use antibiotic-producing bacteria to control garden parasites. Nature 398, 701-704. doi: 10.1038/19519

de Souza, D., Bézier, A., Depoix, D., Drezen, J.-M., and Lenoir, A. (2009). Blochmannia endosymbionts improve colony growth and immune defence in the ant Camponotus fellah. BMC Microbiol. 9:29. doi: 10.1186/1471-2180-9-29

Dietrich, C., Köhler, T., and Brune, A. (2014). The cockroach origin of the termite gut microbiota: patterns in bacterial community structure reflect major evolutionary events. Appl. Environ. Microbiol. 80, 2261-2269. doi: 10.1128/AEM.04206-13

Engel, P., and Moran, N. A. (2013). The gut microbiota of insects - diversity in structure and function. FEMS Microbiol. Rev. 37, 699-735. doi: 10.1111/15746976.12025

Evans, J. D., Aronstein, K., Chen, Y. P., Hetru, C., Imler, J. L., Jiang, H., et al. (2006). Immune pathways and defence mechanisms in honey bees

\section{AUTHOR CONTRIBUTIONS}

$\mathrm{SO}, \mathrm{NB}$, and SHY wrote, edited and contributed equally to the manuscript.

\section{ACKNOWLEDGMENTS}

A special thank you to Michael Poulsen. We also thank Jane de Verges, Sheila Peterson and our reviewers for the valuable discussion, review and comments on the earlier version of this manuscript. SO was supported by a Ph.D. stipend from the Department of Biology, University of Copenhagen, and the Danish National Research Foundation (DNRF57), NB was supported by the Academy of Finland (decision numbers: 251337, 252411, 284666, and 289731) and the University of Helsinki, and SHY was supported by Swiss National Science Foundation to Prof. Michel Chapuisat (grant number: 31003A146641) and the Department of Ecology and Evolution, University of Lausanne.
Apis mellifera. Insect Mol. Biol. 15, 645-656. doi: 10.1111/j.1365-2583.2006. 00682.x

Evison, S. E. F., Fazio, G., Chappell, P., Foley, K., Jensen, A. B., and Hughes, W. O. H. (2013). Host-parasite genotypic interactions in the honey bee: the dynamics of diversity. Ecol. Evol. 3, 2214-2222. doi: 10.1002/ece3.599

Gadau, J., Helmkampf, M., Nygaard, S., Roux, J., Simola, D. F., Smith, C. R., et al. (2012). The genomic impact of 100 million years of social evolution in seven ant species. Trends Genetics 28, 14-21. doi: 10.1016/j.tig.2011.08.005

Grozinger, C. M., and Robinson, G. E. (2015). The power and promise of applying genomics to honey bee health. Curr. Opin. Insect Sci. 10, 124-132. doi: 10.1016/j.cois.2015.03.007

Hamilton, C., Lejeune, B. T., and Rosengaus, R. B. (2011). Trophallaxis and prophylaxis: social immunity in the carpenter ant Camponotus pennysylvanicus. Biol. Lett. 7, 89-92. doi: 10.1098/rsbl.2010.0466

Hoffmann, J. A. (1995). Innate immunity of insects. Curr. Opin. Immunity 7, 4-10. doi: 10.1016/0952-7915(95)80022-0

Hongoh, Y. (2010). Diversity and genomes of uncultured microbial symbionts in the termite gut. Biosci. Biotechnol. Biochem. 74, 1145-1151. doi: 10.1271/bbb.100094

Kapheim, K. M., Pan, H., Li, C., Salzberg, S. L., Puiu, D., Magoc, T., et al. (2015). Social evolution: Genomic signatures of evolutionary transitions from solitary to group living. Science 348, 1139-1143. doi: 10.1126/science.aaa4788

Koch, H., Cisarovsky, G., and Schmid-Hempel, P. (2012). Ecological effects on gut bacterial communities in wild bumblebee colonies. J. Anim. Ecol. 81, 1202-1210. doi: 10.1111/j.1365-2656.2012.02004.x

Koch, H., and Schmid-Hempel, P. (2011). Socially transmitted gut microbiota protect bumble bees against an intestinal parasite. Proc. Natl. Acad. Sci. U.S.A. 108, 19288-19292. doi: 10.1073/pnas.1110474108

Konrad, M., Vyleta, M. L., Theis, F. J., Stock, M., Tragust, S., Klatt, M., et al. (2012). Social transfer of pathogenic fungus promotes active immunisation in ant colonies. PLoS Biol. 10:e1001300. doi: 10.1371/journal.pbio.1001300

Korb, J., Poulsen, M., Hu, H., Li, C., Boomsma, J. J., Zhang, G., et al. (2015). A genomic comparison of two termites with different social complexity. Front. Genet. 6:9. doi: 10.3389/fgene.2015.00009

Kozich, J. J., Westcott, S. L., Baxter, N. T., Highlander, S. K., and Schloss, P. D. (2013). Development of a dual-index sequencing strategy and curation pipeline for analyzing amplicon sequence data on the MiSeq Illumina sequencing platform. Appl. Environ. Microbiol. 79, 5112-5120. doi: 10.1128/AEM. 01043-13

Libbrecht, R., and Keller, L. (2015). The making of eusociality: insights from two bumblebee genomes. Genome Biol. 16:75. doi: 10.1186/s13059-015-0635-z 
López-Uribe, M. M., Sconiers, W. B., Frank, S. D., Dunn, R. R., and Tarpy, D. R. (2016). Reduced cellular immune response in social insect lineages. Biol. Lett. 12:20150984. doi: 10.1098/rsbl.2015.0984

Martinson, V. G., Danforth, B. N., Minckley, R. L., Rueppell, O., Tingek, S., and Moran, N. A. (2011). A simple and distinctive microbiota associated with honey bees and bumble bees. Mol. Ecol. 20, 619-628. doi: 10.1111/j.1365294X.2010.04959.x

Mattila, H. R., Rios, D., Walker-Sperling, V. E., Roeselers, G., and Newton, I. L. G. (2012). Characterization of the active microbiotas associated with honey bees reveals healthier and broader communities when colonies are genetically diverse. PLoS ONE 7:e32962. doi: 10.1371/journal.pone.0032962

Mattoso, T. C., Moreira, D. D. O., and Samuels, R. I. (2011). Symbiotic bacteria on the cuticle of the leaf-cutting ant Acromyrmex subterraneus subterraneus protect workers from attack by entomopathogenic fungi. Biol. Lett. 8, 461-464. doi: 10.1098/rsbl.2011.0963

Meunier, J. (2015). Social immunity and the evolution of group living in insects. Philos. Trans. R. Soc. Lond. B Biol. Sci. 370:20140102. doi: 10.1098/rstb.2014.0102

Moeller, A. H., Foerster, S., Wilson, M. L., Pusey, A. E., Hahn, B. H., and Ochman, H. (2016). Social behavior shapes the chimpanzee pan-microbiome. Sci. Adv. 2:e1500997. doi: 10.1126/sciadv.1500997

Moran, N. A. (2015). Genomics of the honey bee microbiome. Curr. Opin. Insect Sci. 10, 22-28. doi: 10.1016/j.cois.2015.04.003

Nish, S., and Medzhitov, R. (2011). Host defense pathways: role of redundancy and compensation in infectious disease phenotypes. Immunity 34, 629-636. doi: 10.1016/j.immuni.2011.05.009

Otani, S., Mikaelyan, A., Nobre, T., Hansen, L. H., Koné, N. G. A., Sørensen, S. J., et al. (2014). Identifying the core microbial community in the gut of fungus-growing termites. Mol. Ecol. 23, 4631-4644. doi: 10.1111/mec.12874

Otti, O., Tragust, S., and Feldhaar, H. (2014). Unifying external and internal immune defences. Trends Ecol. Evol. 29, 625-634. doi: 10.1016/j.tree.2014.09.002

Poulsen, M., Hu, H., Li, C., Chen, Z., Xu, L., Otani, S., et al. (2014). Complementary symbiont contributions to plant decomposition in a fungus-farming termite. Proc. Natl. Acad. Sci. U.S.A. 111, 14500-14505. doi: 10.1073/pnas.13197 18111

Rosengaus, R. B., Schultheis, K. F., Yalonetskaya, A., Bulmer, M. S., DuComb, W. S., Benson, R. W., et al. (2014). Symbiont-derived beta-1,3-glucanases in a social insect: mutualism beyond nutrition. Front. Microbiol. 5:607. doi: 10.3389/fmicb. 2014.00607

Roux, J., Privman, E., Moretti, S., Daub, J. T., Robinson-Rechavi, M., and Keller, L. (2014). Patterns of positive selection in seven ant genomes. Mol. Biol. Evol. 31, 1661-1685. doi: 10.1093/molbev/msul41

Sabree, Z. L., Huang, C. Y., Arakawa, G., Tokuda, G., Lo, N., Watanabe, H., et al. (2012). Genome shrinkage and loss of nutrient-providing potential in the obligate symbiont of the primitive termite mastotermes darwiniensis. Appl. Environ. Microbiol. 78, 204-210. doi: 10.1128/AEM.06540-11

Sackton, T. B., Werren, J. H., and Clark, A. G. (2013). Characterizing the infectioninduced transcriptome of Nasonia vitripennis reveals a preponderance of taxonomically-restricted immune genes. PLOS ONE 8:e83984. doi: 10.1371/journal.pone.0083984

Sadd, B. M., Barribeau, S. M., Bloch, G., de Graaf, D. C., Dearden, P., Elsik, C. G., et al. (2015). The genomes of two key bumblebee species with primitive eusocial organization. Genome Biol. 16:76. doi: 10.1186/s13059-015-0623-3
Sapountzis, P., Zhukova, M., Hansen, L. H., Sørensen, S. J., Schiøtt, M., and Boomsma, J. J. (2015). Acromyrmex leaf-cutting ants have simple gut microbiota with nitrogen-fixing potential. Appl. Environ. Microbiol. 8, 5527-5537. doi: 10.1128/AEM.00961-15

Schöning, C., Gisder, S., Geiselhardt, S., Kretschmann, I., Bienefeld, K., Hilker, M., et al. (2012). Evidence for damage-dependent hygienic behaviour towards Varroa destructor-parasitised brood in the western honey bee, Apis mellifera. J. Exp. Biol. 215, 264-271. doi: 10.1242/jeb.062562

Simola, D. F., Wissler, L., Donahue, G., Waterhouse, R. M., Helmkampf, M., Roux, J., et al. (2013). Social insect genomes exhibit dramatic evolution in gene composition and regulation while preserving regulatory features linked to sociality. Genome Res. 23, 1235-1247. doi: 10.1101/gr.155408.113

Simone, M., Evans, J. D., and Spivak, M. (2009). Resin collection and social immunity in honey bees. Evolution 63, 3016-3022. doi: 10.1111/j.15585646.2009.00772.x

Smith, C. R., Smith, C. D., Robertson, H. M., Helmkampf, M., Zimin, A., Yandell, M., et al. (2011). Draft genome of the red harvester ant Pogonomyrmex barbatus. Proc. Natl. Acad. Sci. U.S.A. 108, 5667-5672. doi: 10.1073/pnas.1007901108

Terrapon, N., Li, C., Robertson, H. M., Ji, L., Meng, X., Booth, W., et al. (2014). Molecular traces of alternative social organization in a termite genome. Nat. Commun. 5:3636. doi: 10.1038/ncomms4636

Viljakainen, L., Evans, J. D., Hasselmann, M., Rueppell, O., Tingek, S., and Pamilo, P. (2009). Rapid evolution of immune proteins in social insects. Mol. Biol. Evol. 26, 1791-1801. doi: 10.1093/molbev/msp086

Visser, A. A., Kooij, P. W., Debets, A. J. M., Kuyper, T. W., and Aanen, D. K. (2011). Pseudoxylaria as stowaway of the fungus-growing termite nest: interaction asymmetry between Pseudoxylaria, Termitomyces and free-living relatives. Fungal Ecol. 4, 322-332. doi: 10.1016/j.funeco.2011.05.003

Wilson-Rich, N., Spivak, M., Fefferman, N. H., and Starks, P. T. (2009). Genetic, individual, and group facilitation of disease resistance in insect societies. Annu. Rev. Entomol. 54, 405-423. doi: 10.1146/annurev.ento.53.103106.093301

Wong, C. N. A., Ng, P., and Douglas, A. E. (2011). Low-diversity bacterial community in the gut of the fruitfly Drosophila melanogaster. Environ. Microbiol. 13, 1889-1900. doi: 10.1111/j.1462-2920.2011.02511.x

Wurm, Y., and Keller, L. (2010). Parasitoid wasps: from natural history to genomic studies. Curr. Biol. 20, R242-R244. doi: 10.1016/j.cub.2010.01.027

Yek, S. H., Boomsma, J. J., and Schiøtt, M. (2013). Differential gene expression in Acromyrmex leaf-cutting ants after challenges with two fungal pathogens. Mol. Ecol. 22, 2173-2187. doi: 10.1111/mec.12255

Yek, S. H., Nash, D. R., Jensen, A. B., and Boomsma, J. J. (2012). Regulation and specificity of antifungal metapleural gland secretion in leaf-cutting ants. Proc. R. Soc. B 279, 4215-4222. doi: 10.1098/rspb.2012.1458

Conflict of Interest Statement: The authors declare that the research was conducted in the absence of any commercial or financial relationships that could be construed as a potential conflict of interest.

Copyright (c) 2016 Otani, Bos and Yek. This is an open-access article distributed under the terms of the Creative Commons Attribution License (CC BY). The use, distribution or reproduction in other forums is permitted, provided the original author(s) or licensor are credited and that the original publication in this journal is cited, in accordance with accepted academic practice. No use, distribution or reproduction is permitted which does not comply with these terms. 